\title{
A 74 Year Old Woman with Left Shoulder Pain
}

\author{
Jane V. Mayrin, $M D$
}

A 74 year old woman presented to the emergency department complaining of two weeks of left shoulder pain. The pain was sharp, 8 out of 10 in intensity, located in the left shoulder, made worse with breathing, and associated with mild chest discomfort, diaphoresis, and neck pain. There was no radiation and the pain was not worsened with exertion. The patient has a history of Warthin tumor of the left parotid gland and had undergone laryngoscopy and polypectomy two weeks prior to presentation. Around this time she developed the shoulder pain along with overall weakness. At the time of presentation she reported the pain was only present with deep breathing. She denied any shortness of breath, headache, lightheadedness, dizziness, or cough.

In addition to the Warthin tumor, the patient has a history of colon cancer with resection 25 years ago. She has a 50 pack-year smoking history but denied alcohol or substance abuse. She was a retired nurse and lived at home with her husband. Her family history included a father who died in his eighties from a myocardial infarction and a mother who died in her eighties from cancer. She only took zolpidem $10 \mathrm{mg}$ at night as needed for sleep and reported no allergies to medications.

On physical exam she was afebrile at $97.8 \mathrm{~F}$, heart rate 87 beats per minute, respiration rate 18 breaths per minute, blood pressure $117 / 79$, and had an oxygen saturation of $98 \%$ on room air. Her pupils were equal round and reactive to light with normal extraocular movements. The left parotid gland was enlarged. The neck was supple without lymphadenopathy and a jugular venous distension of about $10 \mathrm{~cm}$ was noted. Her heart had a regular rate and rhythm with a II/IV systolic murmur heard best at the left sternal border with no radiation and no third heart sound. Auscultation of the lungs revealed fine crackles at bilateral bases without any wheezing. Her abdominal exam was unremarkable and she had $2+$ pulses in the lower extremities bilaterally without any edema. The left upper extremity had full range of motion without any pinpoint tenderness or effusion.

Laboratory data included hemoglobin of $11.4 \mathrm{~g} / \mathrm{dL}$, WBC count of $8,300 / \mathrm{mm} 3$, platelets of $451,000 / \mathrm{mm} 3$, BNP of $365 \mathrm{pg} / \mathrm{mL}$ and a chemistry panel that was within normal limits. Her first set of Troponin I was $0.23 \mathrm{ng} / \mathrm{mL}$, with the trend of $0.22,0.20,0.15$ (Reference range $<0.05$ to 0.5 ). The EKG showed normal sinus rhythm at $85 \mathrm{bmp}$, with Q waves in leads II, III, and inverted Twave in lead III. Chest $\mathrm{x}$-ray showed bibasilar atelectasis with prominent right paratracheal stripe likely due to vascular congestion without any interstitial edema. Due to the nature of the patient's pain, she had a chest CT performed while in the Emergency Department which showed no pulmonary embolus but was notable for a $3 \mathrm{~cm}$ left ventricular aneurysm of the posterolateral wall at the base of the heart along with a small amount of adjacent pericardial fluid and thickening (Figure 1).

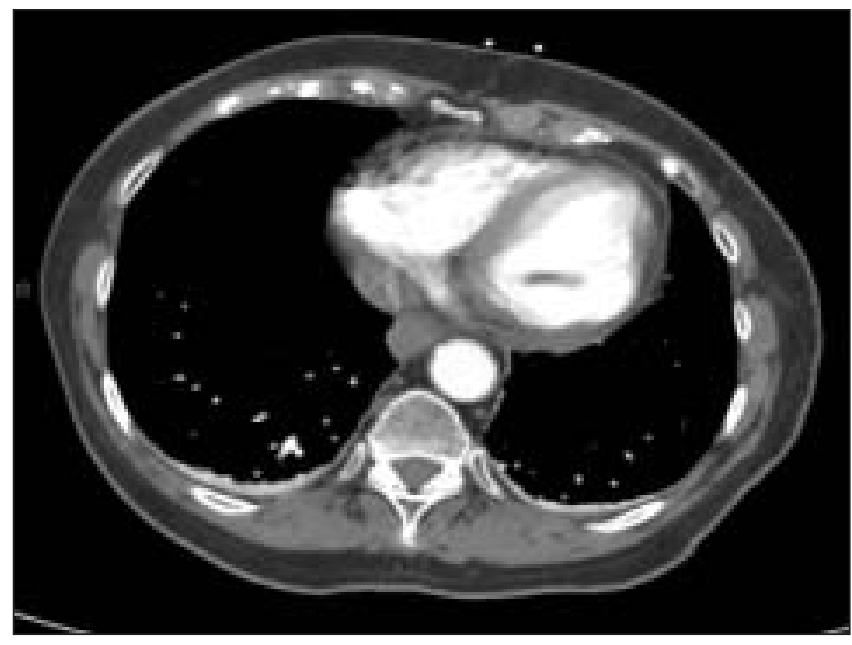

Figure 1. CT chest showing $3 \mathrm{~cm}$ left ventricular aneurysm of the posterolateral wall at the base of the heart.

The patient was admitted to the medicine service for further work up of her pain and to rule out myocardial infarction. Even though her cardiac enzymes were not in a negative range, she ruled out for myocardial infarction with three sets of enzymes within the normal reference range. The night following admission the patient developed rapid atrial fibrillation. Given her baseline hypotension, her rate was controlled with amiodarone, and she was fully anticoagulated with enoxaparin.

The following morning a persantine stress test demonstrated a large inferolateral perfusion defect with partial reversibility at the basal segments indicative of persantine-induced ischemia. The left ventricular ejection fraction was measured as $56 \%$. She also had a transthoracic echocardiogram performed, which was notable for a region of the mid posterior wall with very marked thinning and aneurysmal dilatation. Her left ventricle internal dimensions and wall thickness was normal with overall moderate LV systolic dysfunction with inferior, posterior, and basal-mid lateral akinesis, mild mitral and tricuspid regurgitation and borderline pulmonary hypertension.

Given the findings of the above diagnostic studies, the patient was scheduled to have coronary catheterization the following morning. However, that night the patient was found unresponsive and asystole noted on the cardiac monitor. Despite resuscitative efforts during the PEA code, she never regained a pulse or a blood pressure and died that evening.

Autopsy revealed $500 \mathrm{cc}$ of hemopericardium (Figure 2a and 2b), atherosclerotic cardiovascular disease with thrombotic occlusion of circumflex artery, 50\% occlusion of LAD and RCA, and posterior wall recent myocardial infarction with aneurysmal dilatation. 


\section{Discussion}

There are 3 major mechanical complications of acute myocardial infarction:

- Rupture of the LV free wall;

- Rupture of the interventricular septum;

- Development of mitral regurgitation.

This discussion will focus mostly on the rupture of LV free wall.

\section{Incidence}

Myocardial free wall rupture is the third most common cause of death after myocardial infarction and is 10 times more common than rupture of ventricular septum or papillary muscles. It is a relatively common finding in patients who died of acute myocardial infarction, with some large studies showing cardiac rupture in $14-26 \%$ of these patients. However, of all patients with acute myocardial infarction, the incidence of cardiac rupture is only $1-3 \%$. If thrombolytics are used, death from rupture occurs earlier among these patients and often within 24 hours of drug administration. However, the use of thrombolytics does not increase the incidence of myocardial rupture.

Risk factors for myocardial rupture include the use of thrombolytics, lack of prior history of angina or myocardial infarction, ST-segment elevation or Q wave development on the initial ECG, peak CK-MB above $150 \mathrm{IU} / \mathrm{L}$, anterior location of the infarction, age greater than 70 , and female sex. These associations suggest that the absence of collateral blood flow, as suggested by the lack of previous ischemic symptoms, and the size of the infarct are important determinants of the likelihood of myocardial rupture.

\section{Pathology}

Myocardial rupture occurs within the first 5 days after acute myocardial infarction in about $50 \%$ of cases and within 2 weeks in more than $90 \%$ of cases. It more frequently involves the left

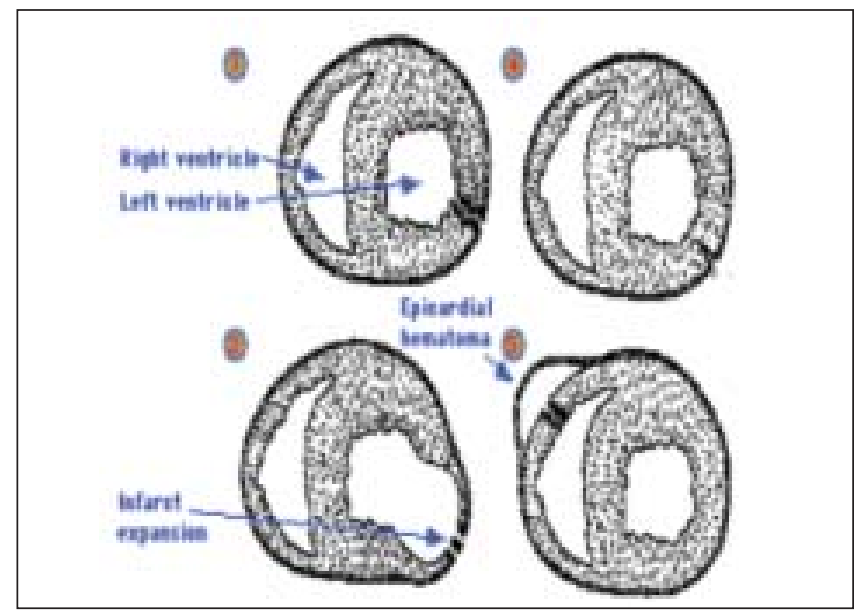

ventricle compared to the right ventricle and rarely involves the atria. The infarct commonly affects the anterior and lateral walls of the left ventricle and the rupture typically occurs near the junction of the infarcted and normal myocardium

\section{Morphologic types of free wall rupture}

1 - normal wall thickness with through and through rupture

2 - expansion of soft necrotic zone with wall rupture

3 - numerous small perforations in the area of myomalacia (multicanalicular rupture)

4 - normal wall thickness with rupture of the outer layer of infarcted area

5 - large epicardial hematoma under pressure

6 - hemorrhagic infarction with grossly intact but abraded and leaking epicardial surface (bleeding infarct)

\section{Evolution of rupture}

Within the first week after the myocardial infarction, neutrophils populate the infarcted area between the necrotic myocytes and produce a zone of coagulative necrosis which is very soft and predisposed to rupture. A myocardial rupture usually occurs at the interface between necrotic and spared myocardium. Once rupture occurs, the pericardium rapidly fills with blood (hemopericardium) and as a result the right ventricle cannot fill and cardiac output rapidly declines.

\section{Pathology of healing}

As mentioned above, the initial phase of acute myocardial infarction is characterized by neutrophil infiltration and myocyte necrosis. The healing phase (after the first week), however, is typified by mononuclear cell and fibroblast infiltration and the absence of polymorphonuclear cells. Resorption of necrotic myocytes often precedes scar formation with accumulation of fibrillar collagen into an increasingly organized network. The complete healing process takes 5- 6 weeks and results in a stable

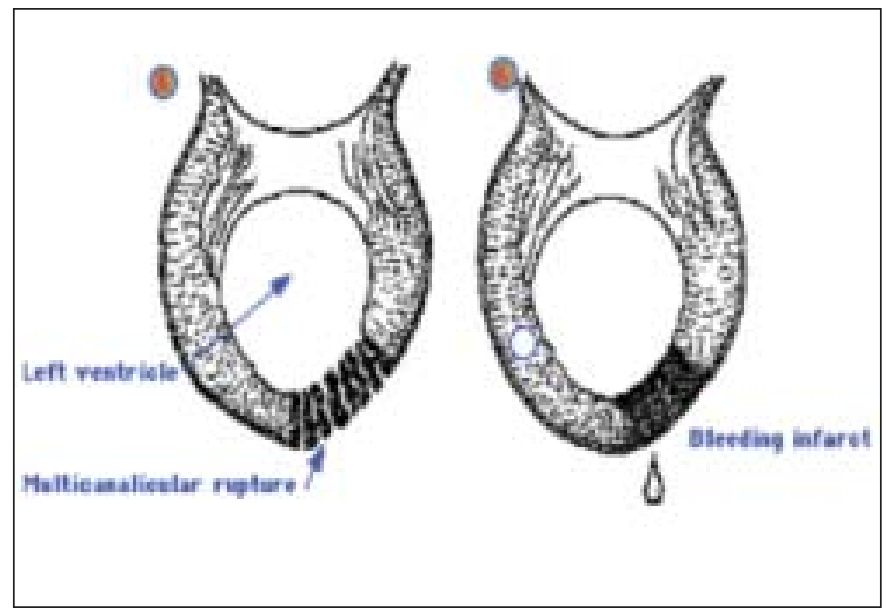




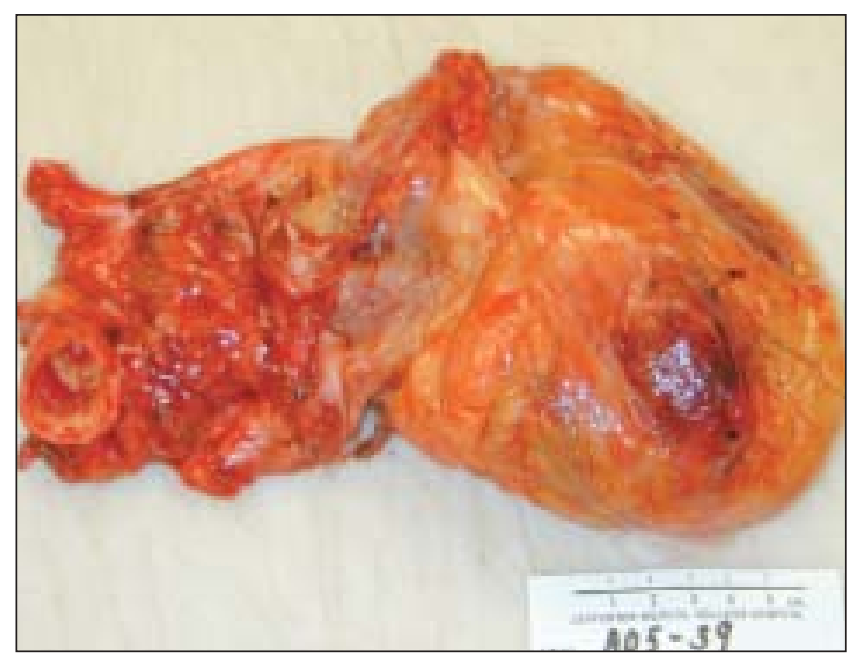

Figure 2a. Posterior wall ventricular aneurysm

scar absent of cellular infiltration. The thinned infarct region is stable and not very vulnerable to mechanical disruption.

\section{New insights into pathology of free wall rupture}

There is a suggestion that defective cardiac remodeling may predispose the heart for rupture. The matrix metalloproteinase (MMP) has been shown to play an important role in cardiac extracellular matrix (ECM) remodeling and cardiac rupture. Over-expression and activities of MMPs induced by TNF (from inflammatory response) at the site of myocardial infarction may increase the degradation of existing collagen in the early stage of infarction, thus contributing to cardiac rupture.

\section{Effects of reperfusion}

It has been shown that rupture is less common in patients with a patent infarct- related coronary artery. Rupture typically occurs in an area that has been infarcted without successful reperfusion. A number of studies has shown that thrombolytic therapy early after myocardial infarction improves survival and decreases risk of myocardial rupture. Late administration of thrombolytics $(6-24 \mathrm{hr}$ after onset of symptoms) does not increase the risk of cardiac rupture, but appears to accelerate the time of onset of rupture events (within $24 \mathrm{hrs}$ of treatment).

\section{Clinical presentation}

Myocardial rupture may present as sudden death in an undetected or silent myocardial infarction. Complete rupture of LV free wall leads to hemopericardium, cardiac tamponade, and subsequent death. Presence of rupture is first suggested by sudden acute right heart failure and shock, which then progresses rapidly to PulselessElectrical-Activity (PEA) codes. During the resuscitative efforts, emergent pericardiocentesis will confirm the diagnosis and transiently relieve the tamponade.

Incomplete rupture occurs when organized thrombus and the pericardium seal the ventricular perforation. This may progress to

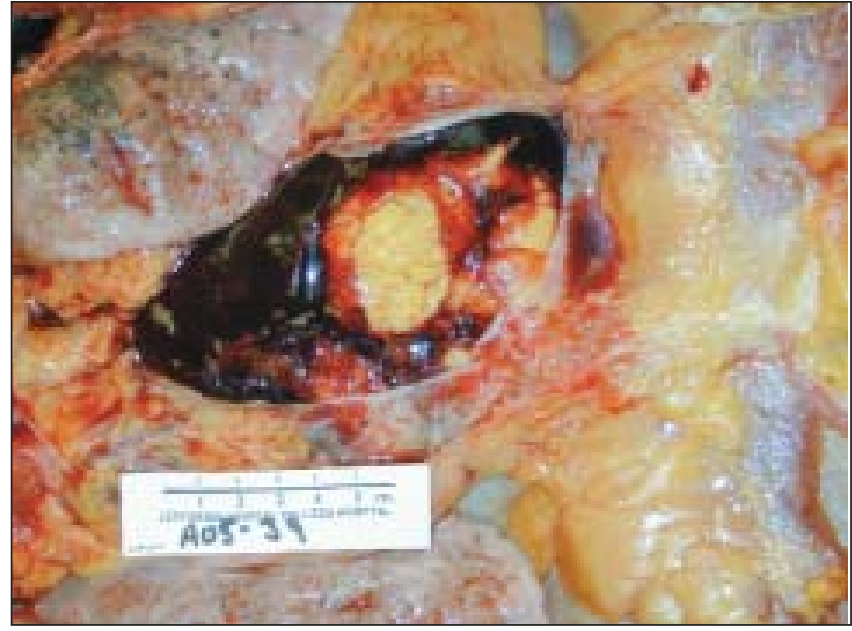

Figure 2b. Hemopericardium on autopsy.

frank rupture with tamponade and death, formation of a false aneurysm walled off by pericardial tissue and communicating with the left ventricle through the perforation, or formation of left ventricular diverticulum. Incomplete rupture may present as recurrent chest pain, nausea, restlessness, abrupt and transient hypotension. It can also cause ECG features mimicking regional pericarditis.

\section{Management}

The survival of patients with free wall myocardial rupture largely depends on rapid recognition and administration of immediate therapy. Bedside echocardiogram and echocardiogram-guided pericardiocentesis should be obtained if the diagnosis of rupture is suspected. If the pericardial fluid includes blood, the patient should immediately proceed to surgery. Fluids, inotropic agents, pressors, and intra-aortic balloon pumps can be used for hemodynamic stabilization while the patient is being transported to the operating room.

\section{References}

1. Laham, RJ. Mechanical Complications of Acute Myocardial Infarction. UpToDate.

2. Exadaktylos, N. et al. Left Ventricular Free Wall Rupture During Acute MI. Early Diagnosis and Treatment. Hellenic J Cardiol. 43: 246-252. 2002

3. Figueras J. Diagnosis, Treatment and Prognosis of Cardiac Rupture in Acute MI. From: http://fac.org.ar/scvc/llave/coronary/figueras

4. Oliva BP, Hammil SC. Cardiac Rupture, a Clinically Predictable Complication of Acute MI: Report of 70 Cases with Clinicopathologic Correlations. J Amer Col Cardiol. 22(3): 720-6, Sep 1993

5. Solomon, S. Renin-Angiotensin System and Cardiac Rupture After Myocardial Infarction. Circulation. 106(17): 2167-2169, Oct 2002

6. Sun, M et al. Excessive Tumor Necrosis Factor Activation After Infarction Contributes to Susceptibility of Myocardial Rupture and Left Ventricular Dysfunction. Circulation. 110(20): 3221-3228, Nov 2004

7. REEDER, G. Identification and Treatment of Complications of Myocardial Infarction. Mayo Clin Proced. 70(9): 880-884, Sep 1995

8. Wehrens $\mathrm{XH}$, Doevendans PA. Cardiac rupture complicating myocardial infarction. Internat J Cardiol. 95 (2-3): 285-92, Jun 2004.

9. UpToDate. 